\title{
Experimental infection of horses with Rickettsia rickettsii
}

\author{
Tatiana Evelyn Hayama Ueno ${ }^{1 *}$, Francisco B. Costa ${ }^{2}$, Jonas Moraes-Filho ${ }^{2}$, Washington Carlos Agostinho ${ }^{2}$, \\ Wilson Roberto Fernandes ${ }^{2}$ and Marcelo B. Labruna ${ }^{2}$
}

\begin{abstract}
Background: Rickettsia rickettsii is vectored by ticks, and some vertebrate hosts can be sources of infection to ticks during bacteremic periods. In Brazil, the main vector for $R$. rickettsii is the tick Amblyomma sculptum, a member of the A. cajennense complex. Horses, in turn, are one of the major hosts for A. sculptum. In this study, horses experimentally infected with $R$. rickettsii were assessed for clinical changes and their capability to transmit the infection to A. sculptum ticks.

Methods: Four horses were infected with $R$. rickettsii through either intraperitoneal injection or infestation with $R$. rickettsii-infected $A$. sculptum ticks. Simultaneously, the animals were infested with non-infected $A$. sculptum ticks. The horses were monitored for 30 days by clinical examination, hematological and biochemical tests, real-time PCR of blood for the detection of Rickettsia, and inoculation of blood in guinea pigs. IgG antibody titers were followed until the horses have shown seronegativity or until the end of the experiment. Uninfected ticks that fed on horses were subjected to real-time PCR and/or were fed on susceptible rabbits.
\end{abstract}

Results: The horses showed no clinical, hematological or blood biochemical alterations, and bacteremia was not detected by real-time PCR or by inoculation of horse blood into guinea pigs. Anti- $R$. rickettsii antibodies were detected in horses from 10 days to 2 years after infection. Uninfected ticks, after feeding on infected horses, showed $2.1 \%$ positivity in real-time PCR, but failed to transmit the infection to rabbits at a next feeding stage.

Conclusions: Rickettsia rickettsii-infected horses did not manifest illness and are not competent amplifier hosts of $R$. rickettsii for $A$. sculptum ticks.

Keywords: Rickettsia rickettsii, Rocky mountain spotted fever, Horses, Ticks, Amblyomma cajennense, Brazil

\section{Background}

The bacterium Rickettsia rickettsii is the etiological agent of a severe illness in humans, known as Brazilian spotted fever (BSF) in Brazil or Rocky Mountain spotted fever in the USA [1]. In Brazil, the disease was first reported during the 1920 s in the state of São Paulo [2], the region with the largest number of notifications throughout the history of this country [3].

In South America, the main vectors of $R$. rickettsii are ticks of the Amblyomma cajennense species complex, also called as $A$. cajennense (sensu lato) (s.l.) $[1,4,5]$. This species complex occurs only in the Americas and is

\footnotetext{
* Correspondence: tatianaueno@apta.sp.gov.br

${ }^{1}$ Agribusiness Technology Agency of São Paulo State, São José do Rio Preto, Brazil

Full list of author information is available at the end of the article
}

distributed from southern USA to northern Argentina [4, 5]. Recently, the A. cajennense species complex was split into six species: A. cajennense (sensu stricto) (s.s.), A. sculptum, A. interandinum, A. mixtum, A. patinoi and A. tonelliae [5]. In the state of São Paulo, southeastern Brazil, this species complex is represented only by A. sculptum, which is therefore, the main vector of $R$. rickettsii in this part of South America $[5,6]$. Hence, all previous studies with $A$. cajennense (s.l.) in southeastern Brazil were in fact related to A. sculptum [6].

Ticks are natural reservoirs of $R$. rickettsii and may remain infected for life. They acquire the infection by transovarial transmission or through feeding on bacteremic vertebrate animals, which are called amplifier hosts [1, 7]. In the case of A. sculptum (reported as $A$. cajennense), the second mechanism is essential for the 
maintenance of the bacterium in the environment because transovarial transmission rate is low and infected ticks have reduced fertility [8]. In the USA, experimental infections showed that several small rodent species were able to transmit the bacterium to Dermacentor andersoni or D. variabilis ticks [9-11]. In Brazil, capybaras (Hydrochoerus hydrochaeris) and opossums (Didelphis aurita) were confirmed as amplifying hosts for A. sculptum ticks (published as $A$. cajennense) $[12,13]$.

Horses are one of the main hosts of all parasitic stages of A. sculptum (reported as A. cajennense) in many BSFendemic regions [14], where high frequencies of $R$. rickettsii-seropositive horses are usually found [15-17]. In previous studies in North America, $R$. rickettsii-experimentally infected horses manifested fever and had rickettsemia for only one day; ticks, however, were not used in these earlier studies [18, 19].

We aimed to investigate the clinical findings in horses experimentally infected with $R$. rickettsii and the role of horses as amplifier hosts of the bacterium for $A$. sculptum ticks.

\section{Methods}

Animals

Four adult crossbred horses were used. Paired serum samples from each animal, taken 15 days apart, proved to be negative through indirect immunofluorescence assay (IFA) [16] (protocol described below) against antigens from six Rickettsia species occurring in Brazil: $R$. rickettsii, R. parkeri, R. amblyommii, R. rhipicephali, $R$. felis and $R$. bellii. During the first 30 days of the experiment (period with artificial tick infestations), horses were housed individually in an isolation stall. After 30 days (at the end of infestations), horses were sprayed with acaricide and transported to a pasture, where they were held until the end of the experiment. Guinea pigs and New Zealand white rabbits were purchased from commercial breeding centre and were housed individually.

\section{Rickettsia rickettsii}

The strain Taiaçu of $R$. rickettsii, originally isolated from the tick $A$. aureolatum in Brazil, was used in this study [20]. This strain has been maintained by consecutive passages in guinea pigs [8] or Vero cells [21]. Because the lineage maintained in guinea pigs probably better preserves the virulence of bacterium, it was used in experimental infections. The Vero cell lineage was used as antigen in IFA.

To prepare the inoculum, guinea pigs previously infected with $R$. rickettsii were euthanized on the third day of fever and then aliquots of spleen, liver, lung and brain were collected and stored at $-80{ }^{\circ} \mathrm{C}$. At the moment of use, the organs were thawed, pooled, macerated with brain heart infusion (BHI), and immediately inoculated in the horses or guinea pigs [8], as described below.

\section{Amblyomma sculptum ticks}

Amblyomma sculptum adult ticks were collected by dragging in Pedreira municipality, a BSF-endemic area of São Paulo, and used to form a laboratory colony. The ticks were identified according to taxonomic key [22] and a recent description/redescription of members of the $A$. cajennense species complex [5]. Uninfected ticks were sequentially fed on rabbits and maintained in an incubator at $25{ }^{\circ} \mathrm{C}$ and a relative humidity of $85 \pm 5 \%$, until obtaining unfed larvae, nymphs and adults. To confirm that the colony was not naturally infected, rabbits used for feeding ticks were tested for antibodies against $R$. rickettsii, $R$. parkeri, $R$. amblyommii, $R$. rhipicephali, $R$. felis and $R$. bellii [16] before and 21 days after infestation, and showed to be negative. Furthermore, rickettsial DNA was not detected by real-time PCR [21] (protocol described below) in female ticks after oviposition.

To produce $R$. rickettsii-infected ticks, part of uninfected larvae were fed on guinea pigs inoculated with $R$. rickettsii. The engorged larvae were collected daily. After molting to nymphs in an incubator, a sample of five ticks corresponding to each detachment day from guinea pigs (total of 60 tested nymphs) was subjected to real-time PCR [21] to verify the infection success. The proportion of positive nymphs was $8.3 \%$, ranging from 0 to $40 \%$ according to detachment day. Only ticks that were collected at days 4 and 5 post-infestation showed positive samples. The nymphs corresponding to these days were fed on new inoculated guinea pigs. The engorged nymphs were collected daily and after molting to adults, a sample of four to eight ticks corresponding to each detachment day from guinea pigs was tested by real-time PCR [21], resulting in 49 tested adult ticks. The infection rate of these adult ticks by real-time PCR was $89.8 \%$ (44/49), ranging from 20 through $100 \%$, according to detachment day. Ticks corresponding to the days with $100 \%$ positive samples were selected for experimental infestations of horses, as described below.

\section{Experimental groups}

The four horses were divided into two groups. In Group 1 (horses 1 and 2), two cotton sleeves for tick infestation were glued to the shaved skin of each horse in the paravertebral region. On day 0,30 males and 30 females of the A. scupltum-infected colony were placed into one of the sleeves. Male ticks were allowed to feed until detachment of the last engorgement female, 24 days after infestation. In the second sleeve, approximately 1,000 larvae, 200 nymphs and 10 adult couples of non-infected 
ticks were placed on day 2 . This infestation was repeated in the same sleeve on days 7, 12, 17 and 22 .

In Group 2 (horses 3 and 4), only one infestation sleeve was attached to each horse. On day 0 each horse was inoculated intraperitoneally with a homogenate of $R$. rickettsii-infected guinea pig organs, as detailed previously [8]. On the same day, approximately 1,000 larvae, 200 nymphs and 10 adult couples of non-infected ticks were placed into the sleeve. This infestation was repeated on days $5,10,15$ and 20.

All horses were clinically examined and their rectal temperatures were measured once a day [23], from 0 through 30 days post-inoculation or post-infestation (dpi). Blood samples were collected from the jugular vein of all horses, every 2 days, from 0 through 30 dpi. Aliquots of blood were distributed as follows: whole blood with ethylenediaminetetraacetic acid (EDTA) for hematological analysis and detection of Rickettsia by real-time PCR [21]; heparinized whole blood for inoculation in guinea pigs; and serum for the detection of anti- $R$. rickettsii antibodies by IFA [16]. Serum biochemical tests were performed on days $0,6,12,18,24$ and 30 . After 30 days of experiment, the blood continued to be collected for monitoring the antibody curve every 7 days until $128 \mathrm{dpi}$, and then at intervals of 7-42 days until the animals became seronegative or the work was ended.

Sera from five time-points were selected to be tested simultaneously against $R$. rickettsii, $R$. parkeri, $R$. amblyommii, $R$. rhipicephali, $R$. bellii and R. felis [16], in order to compare endpoint titers: (i) day 0; (ii) the first day of animal seropositivity to $R$. rickettsii; (iii) the day when titers peaked to $R$. rickettsii; (iv) someday in the decreasing phase of titer; and (v) the last day of seropositivity or animal monitoring.

\section{Hematological and biochemical analyses}

Hematological analysis was performed manually as previously described [24]. Commercial kits were used in the biochemistry tests to quantify serum concentrations of urea, creatinine (DiaSys, Holzheim, Germany), aspartate aminotransferase, total and conjugated bilirubin, total protein, albumin (BioSystems, Barcelona, Spain), and gamma-glutamyl transferase (Randox, Crumlin, Northern Ireland); the readings were done in a LabMax 240 automatic analyzer (Labtest, Lagoa Santa, Brazil). Results were compared with horse reference values $[25,26]$.

\section{Inoculation of horse blood into guinea pigs}

Two guinea pigs were each inoculated intraperitoneally with $500 \mu \mathrm{l}$ of horse blood after each collection. Guinea pigs were clinically monitored and their rectal temperatures were measured up to $21 \mathrm{dpi}$, when they were tested for antibodies against $R$. rickettsii [16]. The animal was considered febrile if temperature exceeded $40{ }^{\circ} \mathrm{C}$ [27].

\section{Evaluation of tick acquisition and transmission of rickettsiae}

In the two horse groups, the sleeve that received uninfected ticks was opened daily for recovering engorged larvae, nymphs and females. Males and non-engorged females that were still attached were removed from horses after $30 \mathrm{dpi}$ and were tested in conjunction to engorged females after oviposition by real-time PCR [21]. Nymphs and adults that molted from engorged larvae and nymphs, respectively, were fed on rabbits. Two rabbits were used for ticks of each horse, the first one receiving ticks that detached from horse up to $15 \mathrm{dpi}$, and the second rabbit receiving ticks that detached from 16 to $30 \mathrm{dpi}$. The rabbits had their rectal temperature measured for 21 days and their serum samples were subjected to IFA [16] to detect anti- $R$. rickettsii antibodies at 0 and 21 dpi. Rabbits were considered febrile if temperature exceeded $40{ }^{\circ} \mathrm{C}$ [27]. After feeding on rabbits, male ticks and females that did not become fully engorged were manually removed and tested by realtime PCR [21]. Engorged nymphs and females recovered from rabbits were also tested by real-time PCR [21] after molting to adults or completing oviposition, respectively.

Only for horse 1, a sample of 90 unfed nymphs and 56 unfed adults, resulting from molting of engorged larvae and nymphs, respectively, was subjected to real-time PCR [21]. The remaining unfed ticks were fed on the rabbits. For the other three horses, all unfed nymphs and adults were placed on rabbits because fewer engorged ticks were recovered from these horses.

Positive ticks in real-time PCR [21] were subjected to conventional PCR $[28,29]$ and sequencing of the Rickettsia ompA gene, as described below [28, 29]. Procedures with ticks, horses and rabbits are summarized in Fig. 1.

\section{Infectivity test of ticks and inocula}

To confirm the viability of rickettsiae in the colony of infected ticks, for each horse in the Group 1 (inoculated via infected ticks), one rabbit was infested simultaneously with five tick couples from this colony. The rabbits were examined daily and their serum samples were subjected to IFA [16] for $R$. rickettsii before and after infestation. To verify whether the ticks were indeed infected, male ticks and females that did not become fully engorged were manually removed from rabbits and horses, and tested by real-time PCR [21]; fully engorged females were tested at the end of oviposition. Among the infected adult ticks that fed on horses, six (three from each horse) were selected for isolation of the bacterium in cell cultures [21,30] and guinea pig inoculation [20]. Isolations were confirmed by sequencing of the rickettsial ompA gene $[28,29]$ from cell cultures and spleen or liver of guinea pigs. 


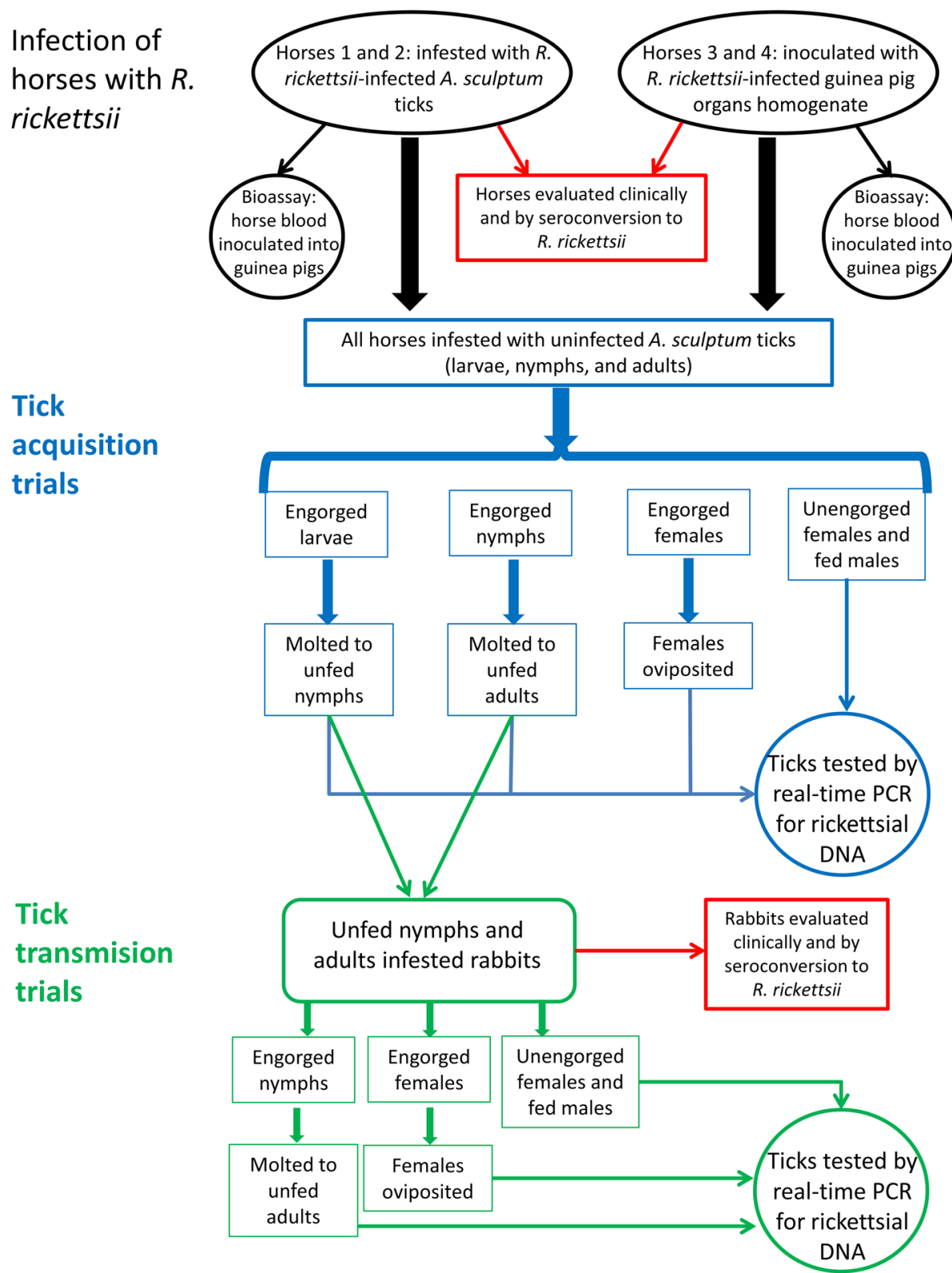

Fig. 1 Diagram illustrating experimental procedures of the present study. Two horses (Group 1) were exposed to rickettsial infection through infestation with Rickettsia rickettsii-infected Amblyomma sculptum ticks. Two other horses (Group 2) were infected through intraperitoneal inoculation of a homogenate of $R$. rickettsii-infected guinea pig organs. The four horses were clinically evaluated and infested with uninfected A. sculptum ticks (larvae, nymphs and adults) during 30 days. Recovered ticks were reared to the next developmental stage and/or tested by real-time PCR for detection of rickettsial DNA. Molted, unfed ticks were allowed to feed on tick-naïve rabbits, which were clinically evaluated for 21 days and tested by seroconversion through testing paired serum samples (days 0 and 21 post-infestation) against $R$. rickettsii antigens. Solely for horse 1, a sample of unfed nymphs and adults, resulting from molting of engorged larvae and nymphs, respectively, was subjected to real-time PCR, and the remaining unfed ticks were fed on the rabbits. For the other three horses, all unfed nymphs and adults were placed on rabbits

For each horse from Group 2 that was intraperitoneally inoculated with $R$. rickettsii, guinea pigs (three guinea pigs for horse 3 and two guinea pigs for horse 4) were simultaneously inoculated with an aliquot of the same homogenate of $R$. rickettsii-infected guinea pig organs that was inoculated into horses, to verify the inoculum viability. Guinea pigs were clinically examined until the 21st dpi, when they were tested for anti- $R$. rickettsii antibodies by IFA [16].

IFA

IFA tests were performed using crude antigens of $R$. rickettsii, $R$. parkeri, $R$. amblyommii, $R$. rhipicephali, $R$. 
bellii and $R$. felis [16]. As secondary antibody, commercial anti-horse IgG, anti-rabbit IgG, or anti-guinea pig IgG antibody (Sigma-Aldrich, St. Louis, MO, USA) was used. Serum samples were initially tested at the 1:64 dilution. Positive samples at initial screening were serially diluted for the determination of endpoint titers. Positive and negative control sera corresponding to each animal species were added to each IFA slide.

\section{PCR}

DNA extraction from horse blood and guinea pig organs was performed with the commercial kit DNeasy Blood \& Tissue Kit (QIAGEN, Hilden, Germany), according to the manufacturer's instructions. The DNA of ticks (processed individually) and cell cultures were extracted by using guanidine isothiocyanate-phenol solution [31]. For detection of the Rickettsia spp. in blood, ticks, cell culture and organs, we used a TaqMan real-time PCR assay targeting a $147 \mathrm{bp}$ fragment of the gltA gene [21]. Each reaction was prepared in a final volume of $25 \mu \mathrm{l}$, containing $2.5 \mu \mathrm{l}$ of $10 \times$ buffer, $2 \mathrm{mM}$ of $\mathrm{MgCl}_{2}, 0.2 \mathrm{mM}$ of each deoxynucleotide triphosphate (dNTP), $0.6 \mu \mathrm{M}$ of each primer, $0.1 \mu \mathrm{M}$ of probe, $0.03 \mathrm{U} / \mu \mathrm{l}$ of Platinum Taq DNA polymerase (Invitrogen, Carlsbad, CA, USA), $2.5 \mu \mathrm{l}$ of DNA template, and $11.65 \mu \mathrm{l}$ of water. Reactions were performed in the 7500 Real Time PCR System apparatus (Applied Biosystems, Foster City, CA, USA) under the following conditions: 1 cycle at $50{ }^{\circ} \mathrm{C}$ for $2 \mathrm{~min}$ and $95{ }^{\circ} \mathrm{C}$ for $10 \mathrm{~min}$, followed by 40 cycles of $95{ }^{\circ} \mathrm{C}$ for $15 \mathrm{~s}$ and $60{ }^{\circ} \mathrm{C}$ for $1 \mathrm{~min}$. In each run, one positive control (DNA of $R$. parkeri strain NOD) and three negative controls (water) were added. Positive samples in real-time PCR were subjected to conventional PCR and sequencing [21] of a fragment of the rickettsial ompA gene for comparison with GenBank sequences and confirmation of the Rickettsia species. The reaction mixture of ompA-based PCR was prepared in a final volume of $25 \mu \mathrm{l}$, containing $2.5 \mu \mathrm{l}$ of $10 \times$ buffer, $2 \mathrm{mM}$ $\mathrm{MgCl}_{2}, 0.25 \mathrm{mM}$ of each dNTP, $0.6 \mu \mathrm{M}$ of primers 190.70 [28] and 190.701 [29], $0.03 \mathrm{U} / \mu \mathrm{l}$ of Platinum Taq DNA polymerase, $2.5 \mu \mathrm{l}$ of DNA template, and $10.85 \mu \mathrm{l}$ of water. The following conditions were used in the Mastercycler Gradient thermocycler (Eppendorf, Hamburg, Germany): 1 cycle at $95{ }^{\circ} \mathrm{C}$ for $5 \mathrm{~min}$, followed by 35 cycles at $95{ }^{\circ} \mathrm{C}$ for $40 \mathrm{~s}, 58{ }^{\circ} \mathrm{C}$ for $30 \mathrm{~s}$, and $65{ }^{\circ} \mathrm{C}$ for $45 \mathrm{~s}$, and a final extension at $72{ }^{\circ} \mathrm{C}$ for $10 \mathrm{~min}$.

Ticks negative in real-time PCR were subjected to conventional PCR targeting mitochondrial $16 S$ rDNA of ticks [32]. The reaction mixture contained the same reagent concentrations used for ompA PCR. The reactions were performed under the following conditions: 1 cycle at $94{ }^{\circ} \mathrm{C}$ for $3 \mathrm{~min}, 10$ cycles of $94{ }^{\circ} \mathrm{C}$ for $30 \mathrm{~s}$, $98{ }^{\circ} \mathrm{C}$ for $30 \mathrm{~s}$, and $72{ }^{\circ} \mathrm{C}$ for $40 \mathrm{~s}, 15$ cycles of $94{ }^{\circ} \mathrm{C}$ for $30 \mathrm{~s}, 50{ }^{\circ} \mathrm{C}$ for $30 \mathrm{~s}$, and $72{ }^{\circ} \mathrm{C}$ for $40 \mathrm{~s}, 10$ cycles of $94{ }^{\circ} \mathrm{C}$ for $30 \mathrm{~s}, 55^{\circ} \mathrm{C}$ for $30 \mathrm{~s}$, and $72{ }^{\circ} \mathrm{C}$ for $40 \mathrm{~s}$, and finally 1 cycle at $72{ }^{\circ} \mathrm{C}$ for $7 \mathrm{~min}$. If the sample was positive, the negative result for the Rickettsia-real-time PCR assay was validated. If the sample was negative, the DNA extraction was considered ineffective and the sample was discarded from the study [8].

\section{Results}

\section{Horse clinical aspects}

All four horses did not show consistent clinical changes. Some clinical and laboratory measurements were slightly different from reference values, but without clinical relevance, as they were observed before and after inoculation or infestation or on sparse days. Rectal temperature values were never above the upper reference limit, although there was a slight increase (temperature over $38^{\circ} \mathrm{C}$ ) from the 3 rd dpi, lasting for a maximum of 3 days (Fig. 2a). Erythrocytes, leukocytes and platelets counts are shown in Fig. 2b-d.

\section{IFA}

Anti- $R$. rickettsii antibodies (titers $\geq 64$ ) were first detected at $10 \mathrm{dpi}$ (horse 3) or $12 \mathrm{dpi}$ (horses 1, 2 and 4). Endpoint titers peaked at 18 to $24 \mathrm{dpi}$ and entered the decreasing curve between 20 and $72 \mathrm{dpi}$. The highest titers were 512 for horse 4, 2,048 for horse 1, and 8,192 for horses 2 and 3 . Horses 1 and 4 remained seropositive until $177 \mathrm{dpi}$ and $254 \mathrm{dpi}$, respectively. The maximum period of antibody persistence could not be determined for horses 2 and 3 because the study was finished before they became seronegative. Horse 2 was monitored until 772 dpi, when the titer was 256 . Horse 3 was monitored up to 366 dpi, when the titer was 256 (Fig. 3).

Comparative tests between antigens showed that all $R$. rickettsii-positive serum samples were also positive for $R$. parkeri, while some of these samples were also positive for the remaining four antigens. Titers for $R$. rickettsii were higher or equal to those for other antigens. On two occasions (on the days of peak titer in horses 1 and 3), the titer for $R$. rickettsii was at least 4-fold higher than the titers for the other five Rickettsia species (Table 1).

\section{Search for Rickettsia in horse blood}

All blood samples from the four horses were negative by real-time PCR throughout the evaluating period. No guinea pigs inoculated with horse blood showed hyperthermia or clinical signs compatible with $R$. rickettsii infection. These guinea pigs were seronegative for $R$. rickettsii at $21 \mathrm{dpi}$.

\section{Tick acquisition and transmission of rickettsiae}

Among the unfed nymphs and adults that had fed on horse 1 as larvae and nymphs, respectively, 2/90 (2.2\%) nymphs and 2/56 (3.6 \%) adults revealed rickettsial DNA by real-time PCR (Table 2 ). 


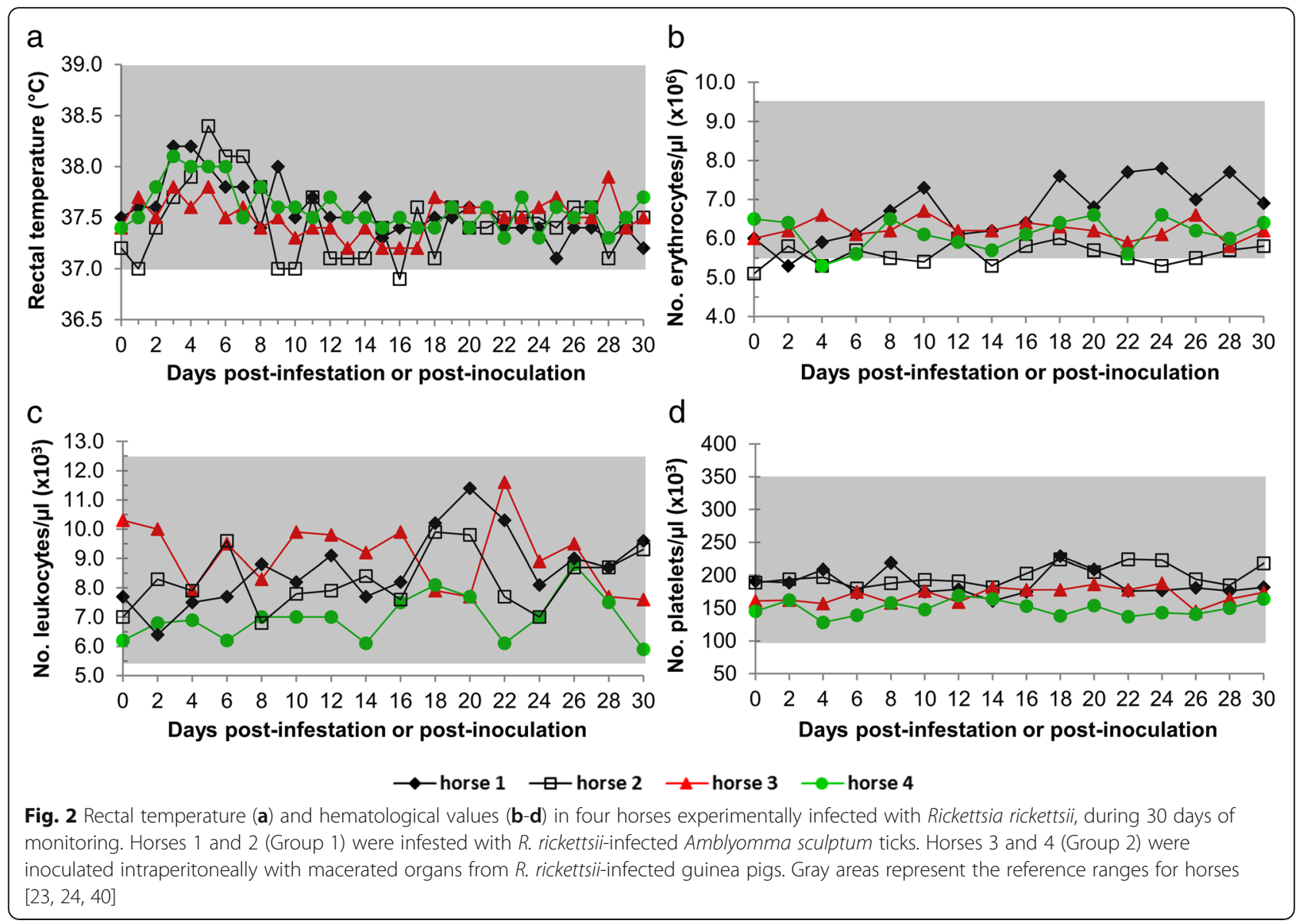

Horse-derived ticks that successfully fed on rabbits consisted of 194 nymphs and 86 adults from horse 1; 77 adults from horse 2; 7 nymphs and 205 adults from horse 3; and 191 nymphs and 73 adults from horse 4. None of the infested rabbits seroconverted to $R$. rickettsii or presented any clinical signs compatible with rickettsiosis.

Considering all acquisition tested ticks, including those fed only on horses and those fed sequentially on horses and rabbits, $2.1 \%(18 / 865)$ were positive by real- time PCR (Table 2). The real-time PCR positivity among ticks that had fed on horses as larvae, nymphs, or adults were $1.1 \%$ (3/275), $0.6 \%$ (3/467) and $9.8 \%(12 / 123)$, respectively. However, when these ticks were subjected to ompA gene-based conventional PCR, just one was positive and its sequencing was faulty due to low DNA concentration. This tick was a nymph that fed as larva on horse 1, and was not fed on rabbit later, so it was not possible to check the transmission capability of this individual tick.

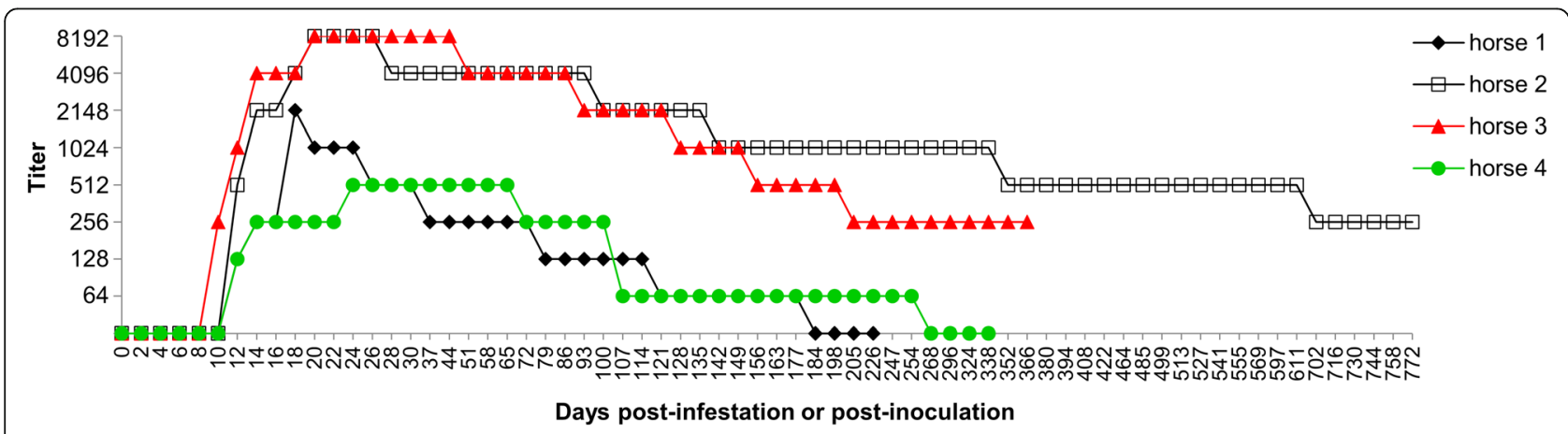

Fig. 3 Antibody titers (IFA $\geq 64$ ) in horses experimentally infected with Rickettsia rickettsii. Abbreviation: IFA, indirect immunofluorescence assay 
Table 1 IFA end-point titers for six Rickettsia species in horses experimentally infected with Rickettsia rickettsii

\begin{tabular}{|c|c|c|c|c|c|c|c|}
\hline \multirow[t]{2}{*}{ Horse } & \multirow[t]{2}{*}{ Days post-infection } & \multicolumn{6}{|c|}{ IFA titers for Rickettsia antigens } \\
\hline & & R. rickettsii & R. parkeri & R. amblyommii & R. rhipicephali & R. bellii & R. felis \\
\hline 1 & 0 & - & - & - & - & - & - \\
\hline 1 & 12 & 128 & 128 & 64 & - & 64 & - \\
\hline 1 & 18 & 2,048 & 256 & 256 & 256 & 256 & 128 \\
\hline 1 & 86 & 128 & 128 & 64 & 64 & 64 & - \\
\hline 1 & 177 & 64 & 64 & - & - & - & - \\
\hline 2 & 0 & - & - & - & - & - & - \\
\hline 2 & 12 & 512 & 256 & 256 & 128 & 256 & - \\
\hline 2 & 20 & 8,192 & 4,096 & 2,048 & 2,048 & 4,096 & 1,024 \\
\hline 2 & 611 & 512 & 256 & - & - & - & - \\
\hline 2 & 772 & 256 & 128 & - & - & - & - \\
\hline 3 & 0 & - & - & - & - & - & - \\
\hline 3 & 10 & 256 & 256 & 64 & 64 & 64 & - \\
\hline 3 & 20 & 8,192 & 2,048 & 1,024 & 2,048 & 512 & 2,048 \\
\hline 3 & 366 & 256 & 128 & 128 & 128 & - & 128 \\
\hline 4 & 0 & - & - & - & - & - & - \\
\hline 4 & 12 & 128 & 64 & - & - & - & - \\
\hline 4 & 24 & 512 & 256 & 128 & 128 & 128 & 256 \\
\hline 4 & 100 & 256 & 128 & 64 & - & - & - \\
\hline 4 & 254 & 64 & 64 & 64 & 64 & 64 & 64 \\
\hline
\end{tabular}

Abbreviations: IFA, indirect immunofluorescence assay-, nonreactive at titer $\geq 64$

\section{Infectivity test of ticks and inocula}

The rabbits that were infested simultaneously to Group 1 horses with $R$. rickettsii-infected ticks, and the guinea pigs that were inoculated with $R$. rickettsii simultaneously to Group 2 horses showed apathy, fever and necrosis of ears and scrotum. One rabbit and two guinea pigs died and their organs tested positive in real-time PCR. All surviving animals were seropositive at $21 \mathrm{dpi}$. All ticks that fed on rabbits were positive by real-time PCR, as were $100 \%(27 / 27)$ and $97 \%(34 / 35)$ of the transmission-fed ticks that fed on horses 1 and 2, respectively. From six ticks selected for rickettsial isolation, $R$. rickettsii was successfully isolated from five of them in cell culture, guinea pig inoculation, or both.

\section{Discussion}

In this study, $R$. rickettsii-infected horses showed no marked clinical changes and the highest rectal temperature observed was $38.4{ }^{\circ} \mathrm{C}$. Rickettsemia was not detected by real-time PCR on horse blood or by guinea pig bioassay. Detection of rickettsia in blood is usually difficult because this bacterium multiplies within endothelial cells, and a low number of these cells are found free in the circulation in cases of mild illness [33]. However, the inoculation of guinea pigs is a sensitive method, as they are very susceptible to $R$. rickettsii, even when receiving low infectious doses [13].
Ricketts [18] and Heinemann \& Moore [19] observed temperatures above $39{ }^{\circ} \mathrm{C}$ lasting for a maximum of 4 days in horses inoculated with USA strains of $R$. rickettsii. In these animals, blood was infective for guinea pigs only at the peak fever day (i.e. the temperature seems to follow the level of bacteremia). Although tick acquisition feeding was not tested in those earlier works, the very short bacteremic period suggests few ticks would acquire the infection. Discrepancies in relation to the occurrence of fever and bacteremia between studies could be attributed to possible differences in virulence of strains and infectious dose.

Among the acquisition feeding ticks that fed on horses, only $2.1 \%$ were positive for Rickettsia through real-time PCR, and among these, only one was positive in conventional PCR targeting the ompA gene. As the real-time PCR is more sensitive than the conventional PCR, this result could be attributed to low bacterium load in ticks, which is corroborated by the high threshold cycle values (more than 31 ) presented by the positive tick samples (data not shown). The absence of illness or seroconversion in rabbits infested with these ticks suggest the rickettsial infection has not been successfully established in ticks, the bacterium did not multiply effectively, and possibly failed to reach the salivary gland. This finding indicates that the horses did not develop a level of rickettsemia sufficient to infect ticks effectively, 
Table 2 Real-time PCR on ticks that were exposed to rickettsiae by feeding on Rickettsia rickettsii-infected horses

\begin{tabular}{|c|c|c|c|c|c|c|c|}
\hline \multirow[t]{2}{*}{ Group } & \multirow[t]{2}{*}{ Horse } & \multicolumn{5}{|c|}{ No. of positive ticks in real-time $P C R /$ no. of tested ticks } & \multirow[t]{2}{*}{ Total } \\
\hline & & $\begin{array}{l}\text { Unfed nymphs (fed as larvae on } \\
\text { horses and molted to nymphs) }\end{array}$ & $\begin{array}{l}\text { Unfed adults (fed as larvae on horses, } \\
\text { fed as nymphs on rabbits, and } \\
\text { molted to adults) }\end{array}$ & $\begin{array}{l}\text { Unfed adults (fed as nymphs on } \\
\text { horses and molted to adults) }\end{array}$ & $\begin{array}{l}\text { Fed adults (fed as nymphs on horses } \\
\text { and fed as adults on rabbits) }\end{array}$ & $\begin{array}{l}\text { Fed adults (fed as adults } \\
\text { on horses) }\end{array}$ & \\
\hline \multirow[t]{2}{*}{ Group $1^{\text {b }}$} & 1 & $2 / 90(2.2 \%)$ & & $2 / 56(3.6 \%)$ & 0/83 (0) & $12 / 51(2.4 \%)$ & $\begin{array}{l}16 / 280 \\
(5.7 \%)\end{array}$ \\
\hline & 2 & & & & $0 / 70(0)$ & $0 / 29(0)$ & $0 / 99(0)$ \\
\hline \multirow[t]{2}{*}{ Group $2^{b}$} & 3 & & $0 / 7(0)$ & & $1 / 189(0.5 \%)$ & $0 / 21(0)$ & $\begin{array}{l}1 / 217 \\
(0.5 \%)\end{array}$ \\
\hline & 4 & & $1 / 178(0.6 \%)$ & & $0 / 69(0)$ & $0 / 22(0)$ & $\begin{array}{l}1 / 269 \\
(0.4 \%)\end{array}$ \\
\hline Total & & $2 / 90(2.2 \%)$ & $1 / 185(0.5 \%)$ & $2 / 56(3.6 \%)$ & $1 / 411(0.2 \%)$ & 12/123 (9.8 \%) & $\begin{array}{l}18 / 865 \\
(2.1 \%)\end{array}$ \\
\hline
\end{tabular}

${ }^{2}$ Engorged females collected from horses or rabbits were tested by real-time PCR after oviposition. Males recovered from horses or rabbits were tested soon after collection

${ }^{b}$ Rickettsial infection in Group 1 (horses 1 and 2) were via Rickettsia rickettsii-infected Amblyomma sculptum ticks, whereas Group 2 (horses 3 and 4) were inoculated intraperitoneally with macerated organs from $R$. rickettsii-infected guinea pigs 
so these arthropods were not able to transmit the bacterium to the next host.

Another aspect that indeed reduces the involvement of horses in the transmission cycle of $R$. rickettsii is the low prolificacy of this vertebrate species, which has a long pregnancy period and usually produces only one foal per pregnancy [34]. As vertebrate hosts develop transient bacteremia and after this period acquire long-lasting immunity, a condition for considering an animal efficient amplifier host is that it gives birth to numerous offspring in a short time, resulting in a continual replacement of susceptible individuals in a particular region [1]. Regarding horses, the only way of abundant replacement would be frequent introduction of individuals from other locations.

All horses developed humoral immune response, which has been reported in several studies that found seropositive horses under natural conditions [15-17]. Earlier studies have already shown indirect evidence of antibody production in $R$. rickettsii-inoculated horses by showing the preventive and curative effect of equine hyperimmune serum on guinea pigs [19, 35]. However, the use of equine serum in human patients would be limited because the protective effect was observed only when the serum was administered during the first 3 days of infection (before the onset of symptoms) and hypersensitivity reactions might occur $[19,35]$. Due to their notable humoral response, horses can be used as efficient sentinels for detecting the circulation of $R$. rickettsii in areas where the primary vector uses horses as hosts, as is the case of $A$. sculptum. The IgG antibody persistence was approximately 6, 26, 12 and 8 months for horses 1, 2, 3 and 4, respectively. Monitoring of horses 2 and 3 was ceased before they became seronegative, i.e. the antibodies probably lasted longer. Other studies followed the animals up to 6 months (dogs and opossums) [36], 10 months (cotton rat Sigmodon hispidus) [37], or 11 months (desert woodrat Neotoma lepida) [38], when the antibodies were still present. Because of the prolonged antibody persistence, the selection of horses to be used as sentinels should preferably include those animals that were born in the target area, or that were living there for over 2 years.

Cross-reactions among $R$. rickettsii and the other five Rickettsia antigens were observed, although no serum elicited a heterologous endpoint titer higher than the homologous titer to $R$. rickettsii. Some studies used the finding of a titer for a specific rickettsia at least 4-fold higher than the titer for any other antigens as criterion to consider this rickettsia, or closely related genotype, the probable responsible for animal infection (homologous antigen) $[16,36]$. Only two sera, corresponding to the peak titer days of two horses, obeyed this criterion. Thus, the determination of homologous antigen in naturally infected horses would probably occur on few occasions and more often when the endpoint titer is high, making other techniques such as cross-absorption [16] necessary to detect which Rickettsia species is involved in the infection.

The infective dose inoculated into horses could not be quantified, although infectivity tests of infected ticks and guinea pig inocula showed both contained viable bacteria pathogenic to rabbits and guinea pigs. The transmission adult ticks that fed on horses 1 and 2 had infection rates of $100 \%$ and $97 \%$, respectively. Considering that each horse from group 1 was infested with 60 ticks that remained in the animals for up to 24 days, it can be inferred that the horses received considerable amounts of bacteria via tick feeding. These results validated our results, i.e. horses did not develop clinical illness due to $R$. rickettsii and did not serve as amplifier hosts of $R$. rickettsii for $A$. sculptum ticks. Finally, our results are also validated by three previous studies that used exactly the same protocols in our laboratory (including the same tick and rickettsial strains), and showed that guinea pigs, capybaras and opossums developed rickettsemia and served as infection sources for $A$. sculptum ticks $[8,12,13]$.

The strain of $R$. rickettsii used in this study was isolated from $A$. aureolatum tick, not from A. sculptum. This strain was used because when the study was initiated there was no Brazilian strain isolated from $A$. sculptum ticks available, which was obtained later [39]. However, the absence of BSF cases in some areas of Brazil where horses are alone the main hosts of $A$. cajennense (s.l.) [1], coupled with the low prolificacy of horses, suggest that another strain would not change the results and reinforce the idea that horses are not amplifier hosts of $R$. rickettsii.

Further studies are needed to evaluate other $R$. rickettsii strains adapted to different populations of $A$. cajennense (s.l.), the role of co-feeding on non-bacteremic hosts in acquisition of $R$. rickettsii by ticks, and the mechanism of immunity against rickettsiae in horses.

\section{Conclusions}

Our results indicate that horses do not have competence to transmit $R$. rickettsii to $A$. sculptum ticks, and that probably they are not relevant in the maintenance cycle of this bacterium in nature. Moreover, $R$. rickettsii-infected horses did not show illness, but present detectable IgG antibodies with high titers and long-lasting persistence.

\section{Acknowledgements}

We thank the Equine Hospital staff of the Faculty of Veterinary Medicine of the University of São Paulo at São Paulo and Pirassununga campi for their help with accommodation and management of horses. 


\section{Funding}

This work was supported by Coordenação de Aperfeiçoamento de Pessoal de Nível Superior (CAPES/PROEX 2327/2015), Brazil.

\section{Availability of data and materials}

Most data generated during this study are included within the article. Supplementary information files are available from the corresponding author upon a reasonable request.

\section{Authors' contributions}

TEHU conceived the study, performed the experiments and wrote the manuscript. FBC participated in the experimental infections of horses and isolation of rickettsiae. JMF participated in the serological tests. WCA participated in the experimental infections of horses and guinea pigs. WRF contributed materials, analysis tools and clinical evaluation of horses. MBL designed and coordinated the study and wrote the manuscript. All authors read and approved the final manuscript.

\section{Consent for publication}

Not applicable.

\section{Ethics approval and consent to participate}

This study was approved by the Ethics Committee of Animal Use of the University of São Paulo (project No. 2101/2010).

\section{Author details}

${ }^{1}$ Agribusiness Technology Agency of São Paulo State, São José do Rio Preto, Brazil. 'Faculty of Veterinary Medicine, University of São Paulo, São Paulo, Brazil.

\section{Received: 21 March 2016 Accepted: 30 August 2016} Published online: 13 September 2016

\section{References}

1. Labruna MB. Ecology of Rickettsia in South America. Ann N Y Acad Sci. 2009;1166:156-66.

2. Piza J, Salles-Gomes L, Meyer J, Fleury JP, Castro O, Rodrigues C, et al. Le typhus exanthématique a São Paulo. C R Seances Soc Biol Fil. 1931;106:1020-2.

3. Ministério da Saúde do Brasil. Sistema de Informação de Agravos de Notificação. Casos confirmados de Febre maculosa. Brasil, Grandes Regiões e Unidades Federadas. 1997 a 2014. http://portalsaude.saude.gov.br/images/ pdf/2014/maio/26/anexo-FebreMaculosa.pdf. Accessed 20 Mar 2015.

4. Estrada-Peña A, Guglielmone AA, Mangold AJ. The distribution and ecological 'preferences' of the tick of humans and other mammals in the Americas. Ann Trop Med Parasitol. 2004;98(3):283-92.

5. Nava S, Beati L, Labruna MB, Cáceres AG, Mangold AJ, Guglielmone AA. Reassessment of the taxonomic status of Amblyomma cajennense (Fabricius, 1787) with the description of three new species, Amblyomma tonelliae $\mathrm{n}$. sp. , Amblyomma interandinum n. sp. and Amblyomma patinoi n. sp., and reinstatement of Amblyomma mixtum, and Amblyomma sculptum (Ixodida: Ixodidae). Ticks Tick Borne Dis. 2014;5(3):252-76.

6. Martins TF, Barbieri ARM, Costa FB, Terassini FA, Camargo LMA, Peterka CRL, et al. Geographical distribution of Amblyomma cajennense (sensu lato) ticks (Parasitiformes: Ixodidae) in Brazil, with description of the nymph of $A$. cajennense (sensu stricto). Parasit Vectors. 2016;9:186.

7. McDade JE, Newhouse VF. Natural history of Rickettsia rickettsii. Annu Rev Microbiol. 1986:40:287-309.

8. Soares JF, Soares HS, Barbieri AM, Labruna MB. Experimental infection of the tick Amblyomma cajennense, Cayenne tick, with Rickettsia rickettsii, the agent of Rocky Mountain spotted fever. Med Vet Entomol. 2012;26(2):139-51.

9. Ricketts HT. Some aspects of Rocky Mountain spotted fever as shown by recent investigations. Med Rec. 1909;76:843-55.

10. Burgdorfer W, Friedhoff KT, Lancaster Jr JL. Natural history of tick-borne spotted fever in the USA: susceptibility of small mammals to virulent Rickettsia rickettsii. Bull World Health Organ. 1966;35(2):149-53.

11. Gage KL, Burgdorfer W, Hopla CE. Hispid cotton rats (Sigmodon hispidus) as a source for infecting immature Dermacentor variabilis (Acari: Ixodidae) with Rickettsia rickettsii. J Med Entomol. 1990;27(4):615-9.

12. Souza CE, Moraes-Filho J, Ogrzewalska M, Uchoa FC, Horta MC, Souza SS, et al. Experimental infection of capybaras Hydrochoerus hydrochaeris by
Rickettsia rickettsii and evaluation of the transmission of the infection to ticks Amblyomma cajennense. Vet Parasitol. 2009;161(1-2):116-21.

13. Horta MC, Moraes-Filho J, Casagrande RA, Saito TB, Rosa SC, Ogrzewalska M, et al. Experimental infection of opossums Didelphis aurita by Rickettsia rickettsii and evaluation of the transmission of the infection to ticks Amblyomma cajennense. Vector Borne Zoonotic Dis. 2009;9(1):109-18.

14. Labruna MB, Kasai N, Ferreira F, Faccini JLH, Gennari SM. Seasonal dynamics of ticks (Acari: Ixodidae) on horses in the state of São Paulo, Brazil. Vet Parasitol. 2002;105(1):65-77.

15. Lemos ER, Machado RD, Coura JR, Guimarães MA, Chagas N. Epidemiological aspects of the Brazilian spotted fever: serological survey of dogs and horses in an endemic area in the State of São Paulo, Brazil. Rev Inst Med Trop Sao Paulo. 1996;38(6):427-30.

16. Horta MC, Labruna MB, Sangioni LA, Vianna MC, Gennari SM, Galvão MA, et al. Prevalence of antibodies to spotted fever group rickettsiae in humans and domestic animals in a Brazilian spotted fever-endemic area in the state of São Paulo, Brazil: serologic evidence for infection by Rickettsia rickettsii and another spotted fever group Rickettsia. Am J Trop Med Hyg. 2004:71(1):93-7.

17. Vianna MC, Horta MC, Sangioni LA, Cortez A, Soares RM, Mafra CL, et al. Rickettsial spotted fever in Capoeirão village, Itabira, Minas Gerais, Brazil. Rev Inst Med Trop Sao Paulo. 2008;50(5):297-301.

18. Ricketts HT. The role of the wood-tick (Dermacentor occidentalis) in Rocky Mountain spotted fever, and the susceptibility of local animals to this disease - a preliminary report. JAMA. 1907;49(1):24-7.

19. Heinemann PG, Moore JJ. Experimental therapy of Rocky Mountain spotted fever: the preventive and curative action of a serum for spotted fever, and the inefficiency of sodium cacodylate as a curative agent for this disease in guinea pigs. J Infect Dis. 1912;10(3):294-304.

20. Pinter A, Labruna MB. Isolation of Rickettsia rickettsii and Rickettsia bellii in cell culture from the tick Amblyomma aureolatum in Brazil. Ann NY Acad Sci. 2006;1078:523-9.

21. Labruna MB, Whitworth T, Horta MC, Bouyer DH, McBride JW, Pinter A, et al. Rickettsia species infecting Amblyomma cooperi ticks from an area in the state of São Paulo, Brazil, where Brazilian spotted fever is endemic. J Clin Microbiol. 2004:42(1):90-8.

22. Barros-Battesti DM, Arzua M, Bechara GH. Carrapatos de importância médico-veterinária da Região Neotropical: um guia ilustrado para identificação de espécies. São Paulo: Vox/International Consortium on Ticks and Tick-borne Diseases (ICTTD-3)/Butantan; 2006.

23. Speirs VC, Wrigley RH. Clinical examination of horses. Philadelphia: WB Saunders; 1997.

24. Feldman BV, Zinkl JG, Jain NC. Schalm's Veterinary Hematology. 5th ed. Philadelphia: Lippincott Williams \& Wilkins; 2000

25. Kaneko JJ, Harvey JW, Bruss ML. Clinical biochemistry of domestic animals. 5th ed. San Diego: Academic; 1997.

26. Meyer DJ, Harvey JW. Veterinary laboratory medicine: interpretation and diagnosis. 3rd ed. St. Louis: WB Saunders; 2004.

27. Hillyer EV, Quesenberry KE. Ferrets, rabbits, and rodents: clinical medicine and surgery. 1st ed. Philadelphia: WB Saunders; 1997.

28. Regnery RL, Spruill CL, Plikaytis BD. Genotypic identification of Rickettsiae and estimation of intraspecies sequence divergence for portions of two rickettsial genes. J Bacteriol. 1991;173(5):1576-89.

29. Roux V, Fournier PE, Raoult D. Differentiation of spotted fever group Rickettsiae by sequencing and analysis of restriction fragment length polymorphism of PCR-amplified DNA of the gene encoding the protein rOmpA. J Clin Microbiol. 1996;34(9):2058-65.

30. Marrero M, Raoult D. Centrifugation-shell vial technique for rapid detection of Mediterranean spotted fever rickettsia in blood culture. Am J Trop Med Hyg. 1989;40(2):197-9.

31. Sangioni LA, Horta MC, Vianna MC, Gennari SM, Soares RM, Galvão MA, et al. Rickettsial infection in animals and Brazilian spotted fever endemicity. Emerg Infect Dis. 2005;11(2):265-70.

32. Black WC, Piesman J. Phylogeny of hard- and soft-tick taxa (Acari: Ixodida) based on mitochondrial 165 rDNA sequences. Proc Natl Acad Sci U S A. 1994;91(21):10034-8.

33. George F, Brouqui P, Boffa MC, Mutin M, Drancourt M, Brisson C, et al. Demonstration of Rickettsia conorii-induced endothelial cell injury in vivo by measuring circulating endothelial cells, thrombomodulin, and von Willebrand fator in patients with Mediterranean spotted fever. Blood. 1993;82(7):2109-16. 
34. Hafez ESE, Hafez B. Reproduction in farm animals. 7th ed. Philadelphia: Lippincott Williams \& Wilkins; 2000.

35. Ricketts HT, Gomez L. Studies on immunity in Rocky Mountain spotted fever: first communication. J Infect Dis. 1908;5(2):221-44.

36. Piranda EM, Faccini JLH, Pinter A, Saito TB, Pacheco RC, Hagiwara MK, et al. Experimental infection of dogs with a Brazilian strain of Rickettsia rickettsii: clinical and laboratory findings. Mem Inst Oswaldo Cruz. 2008;103(7):696-701.

37. Shirai A, Bozeman FM, Humphries JW, Elisberg BL, Faber JE. Experimental infection of the cotton rat Sigmodon hispidus with Rickettsia rickettsii. J Bacteriol. 1967;94(5):1334-9.

38. Lundgren $\mathrm{DL}$, Thorpe BD. Infectious diseases in wild animals in Utah. Experimental infection of rodents with Rickettsia rickettsii. Am J Trop Med Hyg. 1966;15(5):799-806.

39. Krawczak FS, Nieri-Bastos FA, Nunes FP, Soares JF, Moraes-Filho J, Labruna MB. Rickettsial infection in Amblyomma cajennense ticks and capybaras (Hydrochoerus hydrochaeris) in a Brazilian spotted fever-endemic area. Parasit Vectors. 2014;7:7

40. Jain NC. Essentials of veterinary hematology. Philadelphia: Lea \& Febiger; 1993.

Submit your next manuscript to BioMed Central and we will help you at every step:

- We accept pre-submission inquiries

- Our selector tool helps you to find the most relevant journal

- We provide round the clock customer support

- Convenient online submission

- Thorough peer review

- Inclusion in PubMed and all major indexing services

- Maximum visibility for your research

Submit your manuscript at www.biomedcentral.com/submit
Biomed Central 